\title{
Comparison of in vitro susceptibility of Haemophilus influenzae strains to various antimicrobial drugs
}

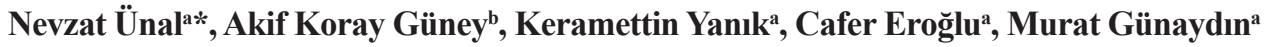 \\ ${ }^{a}$ Department of Medical Microbiology, Medical School, Ondokuz Mayis University, Samsun, Turkey \\ ${ }^{b}$ Microbiology Laboratory, Ankara Atatürk Chest Diseases and Chest Surgery Educational and Research Hospital, Ankara, \\ Turkey
}

\begin{tabular}{|c|c|}
\hline \multicolumn{2}{|c|}{ ARTICLE INFO } \\
\hline \multicolumn{2}{|c|}{ Article History } \\
\hline Received & 17 / 02 / 2013 \\
\hline Accepted & $28 / 03 / 2013$ \\
\hline
\end{tabular}

\section{* Correspondence to:}

Nevzat Ünal

Department of Medical Microbiology,

Medical School,

Ondokuz Mayis University,

Samsun, Turkey

e-mail: drnevzatunal@hotmail.com

\section{Keywords:}

Empirical antibiotic

Haemophilus influenzae

Resistance

Treatment

\section{ABSTRACT}

Haemophilus species are the members of the normal flora of human upper respiratory tract. Haemphilus influenzae is the prominent organism in terms of morbidity and mortality. Haemophilus spp. cause serious infections that can progress to death. The aim of this study is to determine the frequency and antimicrobial susceptibility pattern of $H$. influenzae strains isolated from clinical samples between 2005 and 2010. H. influenzae strains from clinical samples were identified by BBL Crystal AutoReader or Vitek 2 Compact automated system. Antibiotic susceptibility tests were performed according to the standards of CLSI for $H$. influenzae strains. One hundred and fifty-eight $H$. influenzae strains isolated from clinical samples were included in the study. H. influenzae strains were isolated most commonly from samples sent by chest diseases clinics (25.3\%). It was observed that $H$. influenzae was isolated most commonly from lower respiratory tract samples (sputum and tracheal aspirate). In the study period, $29.1 \%$ and $13.9 \%$ of the isolates were found to be resistant to trimethoprim sulfamethoxazole and ampicillin, respectively. Antibiotic resistance rate was $3.7 \%$ for ceftazidime and no resistance was detected for meropenem, imipenem and cefuroxime. We found a statistically significant difference in the resistance to ampicillin and trimethoprim sulfamethoxazole between 2005 and $2010(p<0.05)$. But, there was no significant difference in the resistance to ceftazidime, chloramphenicol, aztreonam and rifampicin over the years $(\mathrm{p}>0.05)$. We suggest that trimethoprim-sulfamethoxazole and ampicillin are not appropriate options for the treatment of infections caused by H. influenzae in our hospital. Second generation cephalosporins and quinolones can be used for this purpose. We suggest that determination of the frequency of $H$. influenzae from clinical samples and its antibiotic susceptibility pattern may produce data for epidemiological studies and guide empirical antibiotic therapy.

J. Exp. Clin. Med., 2013; 30:165-169

(C) 2013 OMU

\section{Introduction}

Haemophilus influenzae is a non-spore forming gram negative rod that can only live in human being and has no other known natural host. The organism may be found in normal upper respiratory tract flora of children and adults. It may also colonize in nose and conjuctiva, and rarely genital tract. Human upper respiratory tract is colonized by non-typeable strains and less frequently by typeable strains including $H$. influenzae type $\mathrm{b}$ (Hib). While non-typeable strains can cause otitis media, acute exacerbation of chronic obstructive pulmonary disease, pneumonia, sinusitis, and sepsis, $H$. influenzae type b causes serious infections such as meningitis, epiglottitis and septic arthritis (Murphy, 2009). H. influenzae was one of the three major causes of bacterial meningitis in many countries until the implementation of widespread vaccination programs. Immunocompromised patients and children under three years of age who do not have adequate antibody levels have a high risk of infections caused by $H$. influenzae (Kilian, 2007). Hib is a significant pathogen for children and accounts for $95 \%$ of invasive $H$. influenzae infections. In particular, the organism is one of the most frequently isolated bacteria from meningitis at the age of 0-2 (Eşel et al., 2000). Unencapsulated strains cause infections mostly in adults with an underlying disease such as chron- 
ic obstructive pulmonary disease or cystic fibrosis. The association of pneumonias primarily caused by $H$. influenzae with bacteremias has become increasingly important (Kilian, 2007). In addition to this increase, it is reported that there is also an increase in the ampicillin resistance. As well as the early diagnosis and management of these cases, more serious infections and potential complications can be prevented by appropriate empirical treatment. However, a great number of clinical isolates are resistant to $\beta$-lactam antibiotics, particularly ampicillin, chloramphenicol and tetracycline, basically due to dissemination of conjugative plasmids. The rate of ampicillin resistance of $H$. influenzae isolates ranges from $5 \%$ to $60 \%$ in different countries (Berkiten, 2004). Therefore, it is important to know local antibacterial resistance patterns to start appropriate emprical treatment.

In the study, we aimed to determine the distribution and antimicrobial susceptibility pattern of $H$. influenzae strains isolated from various clinical samples sent to our laboratory.

\section{Materials and methods}

$H$. influenzae strains isolated from various clinical samples sent to our laboratory from various clinics between 2005 and 2010 were included in the study. Samples admitted to our laboratory were inoculated on 5\% sheep blood agar, eosin methylene blue (EMB) agar and chocolate agar. Following the incubation of culture plates at $37^{\circ} \mathrm{C}$ for 18 to 24 hours, organisms were evaluated in terms of colonial morphology and staining characteristics. Suspected H. influenzae colonies were identified by BBL Crystal autoReader (BD Diagnostic Systems USA) or Vitek 2 Compact automated system (bioMerieux SA France). A total of $158 \mathrm{H}$. influenzae strains were included in the study, duplicate isolates from one patient were removed. Antibiotic susceptibility tests were performed by Kirby-Bauer disc diffusion method according to the recommendations of Clinical and Laboratory Standards Institute (CLSI). H. influenzae ATCC 49247 and H. influenzae ATCC 49766 were used as the internal quality control strains. Statistical analysis was performed by using SPSS (Statistical Package for Social Scienses) 18.0 statistical program. Univariate chi-squared analysis was used to evaluate annual trends in antibiotic resistance.

\section{Results}

A total of $158 \mathrm{H}$. influenzae strains isolated from samples sent to Diagnostic Bacteriology Laboratory in the study period were included in the study. Gram stain of Haemophilus influenzae is shown in Figure 1. Growth of Haemophilus influenzae on chocolate agar is shown in Figure 2. Antibiotic susceptibility tests are shown in Figure 3 on the Haemophilus test medium (HTM).

H. influenzae strains were most frequently isolated from the samples from chest diseases clinic and secondly from internal medicine clinic (Table 1). H. influenzae was most frequently isolated from sputum samples. Clinical sample distribution (Table 2) and resistance pattern of isolated strains by years (Table 3 ) are given. Accordingly, there was a statistically significant difference between resistance to ampicillin and trimethoprim-sulfamethoxazole between 2005 and 2010 $(p<0.05)$. But, there was no significant difference among resistance to ceftazidime, chloramphenicol, aztreonam and rifampicin by years $(\mathrm{p}>0.05)$.

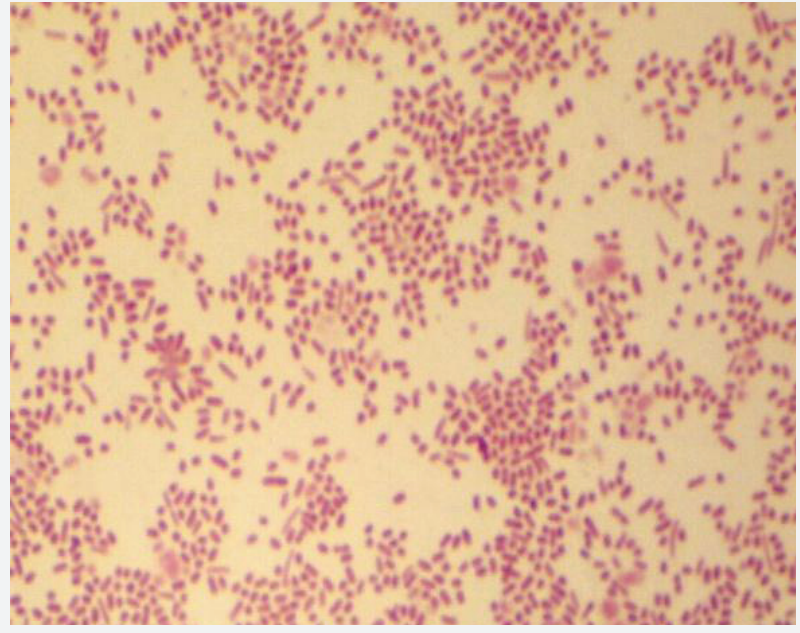

Fig. 1. Gram stain of Haemophilus influenzae

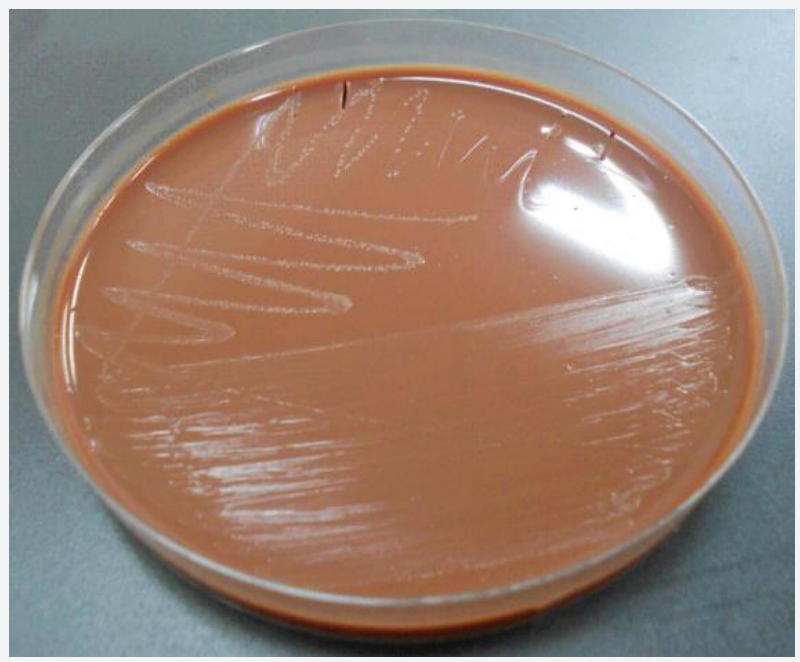

Fig. 2. Growth of Haemophilus influenzae on chocolate agar

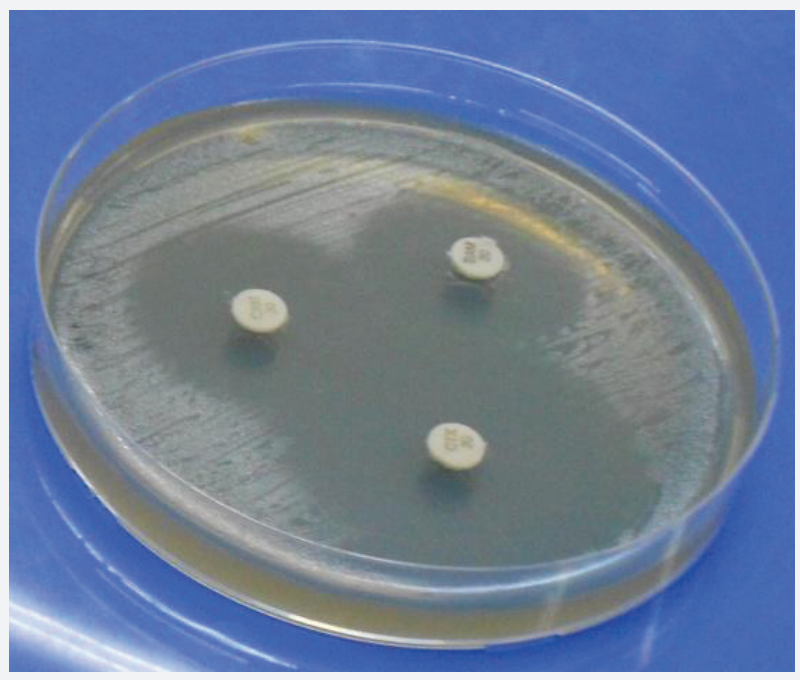

Fig. 3. The antimicrobial susceptibility of Haemophilus influenzae strain on Haemophilus test medium 


\section{Discussion}

$H$. influenzae is an important pathogen that is commonly isolated from many parts of the world and can cause high morbidity and mortality (Tristram et al., 2007). It is important to know local resistance pattern in order to give an effective treatment. However, resistance rates vary among geographical regions. When studies from Turkey were examined; in the study by Şenol and Eriş (2000) the resistance rates of all isolates to ampicillin, erythromycin, ciprofloxacin, trimethoprim-sulfamethoxazole, tetracycline and chloramphenicol were found $46 \%, 48 \%, 16 \%, 36 \%, 31 \%$ and $13 \%$ respectively. In the study by İlki et al. (2010) between 2003 and 2006 , resistance rates of $H$. influenzae isolates to ampicillin, trimethoprim-sulfamethoxazole, chloramphenicol and cefotaxime were found $3.3 \%, 25.5 \%, 2.2 \%$ and $0 \%$ respectively. In the study by Uncu et al. (2007) between 2005 and 2006, the authors found resistance rates of $H$. influenzae isolates to ampicillin, trimethoprim-sulfamethoxazole, chlorampheni-

\begin{tabular}{lc}
$\begin{array}{l}\text { Table 1. Distribution of samples from which } H \text {. influenzae was } \\
\text { isolated according to clinics }\end{array}$ \\
\hline Clinics & Number of samples \\
\hline Chest diseases & 40 \\
Internal medicine & 36 \\
Pediatrics & 18 \\
Intensive care unit & 15 \\
Cardiology & 12 \\
Emergency & 9 \\
Oncology & 6 \\
Chest surgery & 5 \\
Neurosurgery & 4 \\
Infectious diseases & 3 \\
Others & 10 \\
\hline
\end{tabular}

Table 2. Distribution of clinical samples from which $H$. influenzae was isolated according to years

\begin{tabular}{lccccccc} 
Sample & $\mathbf{2 0 0 5}$ & $\mathbf{2 0 0 6}$ & $\mathbf{2 0 0 7}$ & $\mathbf{2 0 0 8}$ & $\mathbf{2 0 0 9}$ & $\mathbf{2 0 1 0}$ & Total \\
\hline Sputum & 12 & 8 & 33 & 24 & 20 & 11 & 108 \\
Tracheal aspirate & 0 & 4 & 6 & 5 & 7 & 3 & 25 \\
Conjuctiva & 1 & 2 & 5 & 6 & 1 & 1 & 16 \\
Cerebrospinal & 0 & 0 & 0 & 0 & 1 & 1 & 2 \\
Fluid & 1 & 1 & 3 & 0 & 1 & 1 & 7 \\
Others & 14 & 15 & 47 & 35 & 30 & 17 & 158 \\
Total & & & & & &
\end{tabular}

col, tetracycline, imipenem and cephalosporins 3.2\%, 25\%, $6.4 \%, 0 \%, 0 \%, 0 \%$ respectively. In a multicenter study, including 379 clinical isolates, by Şener et al. (2007) between 2004 and 2005, the rates of resistance to ampicillin, ofloxacin, tetracycline and trimethoprim-sulfamethoxazole were found $4.7 \%, 0 \%, 8.5 \%$ and $22.9 \%$, respectively. While ampicillin resistance wasn't detected in some centers in the study, it was over $10 \%$ for many hospitals. In the study, including 861 clinical islates, by Altun and Gür (2008) from Ankara between 2002 and 2007, the rates of resistance to ampicillin, trimethoprim-sulfamethoxazole, ciprofloxacin, meropenem and tetracycline were found $7.2 \%, 23 \%, 0 \%, 0 \%$ and $1.1 \%$, respectively. According to the limited number of studies, ampicillin resistance in Turkey is between $0 \%$ and $52.5 \%$ (Şener et al., 2007). When we examine some of the studies abroad, ampicillin resistance varies between 5\% and $60 \%$ according to countries. Ampicillin and trimethoprim-sulfamethoxazole are excluded from the treatment due to changing or increasing resistance rates. Quinolone resistance is still rare (Kilian, 2007). In the study by Tristam et al. (2007) resistance rates of $H$. influenzae isolates to ampicillin, trimethoprim-sulfamethoxazole, cefuroxime, chloramphenicol and ciprofloxacin were found $17 \%, 17 \%, 0.7 \%, 1.9 \%$ and $0 \%$, respectively. In the study by Ladhani et al. (2008) which investigated a period of twenty years in England, the authors found the resisitance rates to ampicillin, chloramphenicol and tetracyclin as $16.2 \%, 1.2 \%$ and $1.8 \%$, respectively. In the study by Giufre et al. (2011) the resistance rates in H.influenzae to ampicillin, chloramphenicol, ciprofloxacin, cefotaxime and imipenem were found as $10.3 \%, 2.6 \%, 0 \%, 0 \%$ and $2.6 \%$, respectively. In the study by Perez-Trallero et al. (2010) the authors found the resistance rates to ampicillin, cefuroxime, cefotaxime and ciprofloxacin as $13.3 \%, 0.1 \%, 0 \%$ and $0.2 \%$ respectively.

In the study by Cardines et al. (2010) the authors found the resistance rates to ampicillin, amoxicillin clavulanate, cefixime, cefotaxime, imipenem, ciprofloxacin, azithromycin and chloramphenicol in 79 H.influenzae strains isolated from patients with cystic fibrosis between 2004 and 2009 as $13.9 \%, 10.1 \%, 10.1 \%, 0 \%, 17.7 \%, 0 \%, 10.1 \%$ and $3.8 \%$, respectively. In our study, carbapenem resistance was not detected.

In the study by Landhani et al. (2008) from England, the authors investigated a period of twenty years. While ampicillin resistance increased gradually from 1985 until the mid-

\begin{tabular}{|c|c|c|c|c|c|c|c|c|c|c|c|}
\hline Year/Antibiotic $(\mathrm{n}, \%)$ & AMP & SXT & CAZ & $\mathrm{C}$ & MEM & IMP & CXM & AZT & TE & CIP & RA \\
\hline $\begin{array}{l}2005 \\
\quad 14,(8.8)\end{array}$ & $1(7.1)$ & $4(28.5)$ & $1(7.1)$ & 0 & 0 & 0 & 0 & $1(7.1)$ & 0 & 0 & $1(7.1)$ \\
\hline $\begin{array}{l}2006 \\
\quad 15,(9.4)\end{array}$ & $1(6.6)$ & $1(6.6)$ & 0 & 0 & 0 & 0 & 0 & 0 & 0 & 0 & 0 \\
\hline $\begin{array}{l}2007 \\
\quad 47,(29.7)\end{array}$ & $5(10.6)$ & $10(21.2)$ & $1(2.1)$ & 0 & 0 & 0 & 0 & $2(4.2)$ & $4(8.5)$ & 0 & $1(2.1)$ \\
\hline $\begin{array}{l}2008 \\
\quad 35,(22.1)\end{array}$ & $6(17.1)$ & $12(34.2)$ & $3(8.5)$ & $2(5.7)$ & 0 & 0 & 0 & 0 & 0 & $1(2.8)$ & 0 \\
\hline $\begin{array}{l}2009 \\
30,(18.9)\end{array}$ & $9(30)$ & $12(40)$ & 0 & $1(3.3)$ & 0 & 0 & 0 & 0 & 0 & 0 & 0 \\
\hline $\begin{array}{l}2010 \\
\quad 17,(10.7)\end{array}$ & 0 & $7(41.1)$ & $1(5.8)$ & 0 & 0 & 0 & 0 & 0 & 0 & 0 & 0 \\
\hline $\begin{array}{l}\text { Total } \\
\quad 158\end{array}$ & $22(13.9)$ & $46(29.1)$ & $6(3.7)$ & $3(1.8)$ & 0 & 0 & 0 & $3(1.8)$ & $4(2.5)$ & $1(0.6)$ & $2(1.2)$ \\
\hline $\mathrm{P}$ & 0.000 & 0.000 & 0.228 & 0.317 & & & & 0.366 & & 0.096 & \\
\hline
\end{tabular}

AMP: Ampicillin; SXT: Trimethoprim sulfamethoxazole; CAZ: Ceftazidime; C: Chloramphenicol; MEM: Meropenem; IMP: Imipenem; CXM: Cefuroxim; AZT: Aztreonam; TE: Tetracycline; CIP: Ciprofloxacin; RA: Rifampicin; NOTEb: Cefuroxime was not performed for the strains resistant to ceftazidime 
1990 s, it declined to the lowest ratio of $11.6 \%$ in 2004 . While trimethoprim-sulfamethoxazole resistance was $10 \%$ in 1985 , it decreased until 1990 then increased gradually to $11.9 \%$ in 2004. In the study of 578 isolates by Jansen et al. (2006) amoxicillin resistance was reported $19.8 \%, 23.3 \%$ and $16.4 \%$ for $1997 / 98,2002 / 03$ and 2004/05, respectively. In the study by Doern et al. (1997) from USA that included 1537 clinical isolates between 1994 and 1995, ampicillin and trimethoprimsulfamethoxazole resistance were reported $38.9 \%$ and $9 \%$, respectively. In the study by Harrison et al. (2009) from USA that included 143 clinical isolates between 2005 and 2007, amoxicillin and trimethoprim-sulfamethoxazole resistances were reported $32 \%$ and $26.6 \%$, respectively. In the study by Cobos et al. (2008) from Spain that included 197 clinical isolates between 1997 and 2007, there was a gradual decline in the amoxicillin resistance from $38.6 \%$ to $30.2 \%$ and trimethoprim-sulfamethoxazole resistance from $50 \%$ to $34.9 \%$. In our study, $H$. influenzae was most commonly isolated from lower respiratory tract samples (sputum and tracheal aspirate). Resistance of clinical isolates to ampicillin (13.9\%) and trimethoprim-sulfamethoxazole (29.1\%) was found higher compared to other antibiotics. Results were consistent with literature data. In our study, while ampicillin resistance increased between 2005 and 2009, the resistance wasn't seen in the strains isolated in 2010. The change in these years was statistically different $(p<0.05)$. The absence of ampicillin resistance in 2010 may be due to the antibiotic policies in our hospital, but the small number of isolates should be considered. Trimethoprim-sulfamethoxazole resistance decreased in 2006, whereas it increased in the other years. Increase and change in the trimethoprim-sulfamethoxazole resistance in years were found statistically significant $(p<0.05)$. Because cefuroxime wasn't performed for the isolates which was resistant to ceftazidime, cefuroxime susceptibility couldn't determined in these strains. There were no imipenem and meropenem resistance. For tetracycline, chloramphenicol, aztreonam and rifampicin, very low level of resistance was detected in some years.

Minimal resistance to quinolones has been reported in the studies from Turkey and abroad. In the studies from Turkey, it is seen that very different results, sometimes even different from expected, were obtained. This situation may be due to geographical differences, patient groups, methodologies and knowledge and experience of the authors.

Considering our resistance rates, in the empirical treatment of the patients with lower respiratory tract infection, we suggest that trimethoprim-sulfamethoxazole and ampicillin are not viable options. Cefuroxime, ceftazidime, and ciprofloxacin are among the options that can be used firstly for this purpose. We suggest that it is important to determine the frequency of isolation and antibiotic resistance pattern of $H$. influenzae periodically in terms of providing data to epidemiological studies and guiding empirical antibiotic treatment.

\section{REFERENCES}

Altun, B., Gür, D., 2008. Antimicrobial resistance in Haemophilus influenzae strains isolated from various clinical samples in Hacettepe University Children's Hospital (2002-2007). Çocuk Enf. Derg. 2, 50-54.

Berkiten, R., 2004. Beta-Lactamase activity and ampicillin resistance in Haemophilus influenzae strains isolated in Turkey (1987-2002). Ankem Derg. 18, 53-60.

Cardines, R., Giufrè, M., Pompilio, A., Fiscarelli, E., Ricciotti, G., Bonaventura, G.D., Cerquetti, M., 2012. Haemophilus influenzae in children with cystic fibrosis: Antimicrobial susceptibility, molecular epidemiology, distribution of adhesins and biofilm formation. Int. J. Med. Microbiol. 302, 45-52.

Cobos, S.G., Campos, J., Cercenado, Román F, Lázaro, E., Pérez-Vázquez, M., de Abajo, F, Oteo, J.E., 2008. Antibiotic resistance in Haemophilus influenzae decreased, except for-Lactamase-Negative Amoxicillin-Resistant isolates, in parallel with community antibiotic consumption in Spain from 1997 to 2007. Antimicrob. Agents Chemother. 52, 2760-2766.

Doern, G.V., Brueggemann, A.B., P1erce, G., Holley, H.P., Rauch A., 1997. Antibiotic Resistance among clinical isolates of Haemophilus influenzae in the United States in 1994 and 1995 and detection of b-Lactamase-Positive strains Resistant to Amoxicillin-Clavulanate: Results of a national multicenter surveillance study. Antimicrob. Agents Chemother. 292-297.

Eşel, D., Karaca, N., Sümerkan, B., 2000. Antibiotic susceptibilities of Haemophilus influenzae strains isolated from clinical specimens. Ankem Derg. 14, 555.

Giufrè, M., Cardines, R., Caporali, M.G., Accogli, M., D’Ancona, F., Cerquetti, M., 2011. Ten years of Hib vaccination in Italy: Prevalence of non-encapsulated Haemophilus influenzae among invasive isolates and the possible impact on antibiotic resistance. Vaccine. 29, $3857-3862$.

Harrison, C.J., Woods, C., Stout, G., Martin, B., Selvarangan, R., 2009. Susceptibilities of Haemophilus influenzae, Streptococcus pneumoniae, including serotype 19A, and Moraxella catarrhalis paediatric isolates from 2005 to 2007 to commonly used antibiotics. J. Antimicrob. Chemother. 63, 511-519.

Ilki, A., Sağiroğlu, P., Elgörmüş, N., Söyletir, G., 2010. Trends in antibiotic susceptibility patterns of Streptococcus pneumoniae and Haemophilus influenzae isolates: Four years follow up. Mikrobiyol. Bul. 44, 169-175.

Jansen, W.T.M., Verel, A., Beitsma, M., Verhoef, J., Milatovic, D., 2006. Longitudinal European surveillance study of antibiotic resistance of Haemophilus influenzae. J. Antimicrob. Chemother. 58, 873-877.

Kilian, M., Haemophilus. 2007. In Patrick R., Murray, Ellen Jo Baron, James H. Jorgensen, Marie Louise Landry, Michael A., Pfaller (eds). 9th ed. Manual of Clinical Microbiology. ASM Press 1752 N St. N.W. 41, 636-648.

Ladhani, S., Heath, P.T., Ramsay, M.E., Slack, M.P.E., 2008. Changes in antibiotic resistance rates of invasive Haemophilus influenzae isolates in England and Wales over the last 20 years. J. Antimicrob. Chemother. 62, 776-779.

Murphy, T.F., 2009. Haemophilus infections, in Mandell GL, Bennett JE, Dolin R (eds). 7th ed. Mandell, Douglas and Bennett's principles and practice of infectious diseases. Philadelphia: Elsevier, Churchill, Livingstone. 225, 2911-2919.

Perez-Trallero, E., Martín-Herrero, J.E., Mazon, A., García-Delafuente, C., Robles, P., Iriarte, V., Dal-Re', R., García-de-Lomas, J., 2010. Antimicrobial resistance among respiratory pathogens in Spain: Latest data and changes over 11 Years (1996-1997 to 2006-2007). Antimicrob. Agents Chemother. 54, 2953.

Sener, B., Tunçkanat, F., Ulusoy, S., Tünger, A., Söyletir, G., Mülazimoğlu, L., Gürler, N., Oksüz, L., Köksal, I., Aydin, K., Yalçin, A.N., Oğünç, D., Acar, A., Sievers, J., 2007. A survey of antibiotic resistance in Streptococcus pneumoniae and Haemophilus influenzae in Turkey, 2004- 
2005. J. Antimicrob. Chemother. 60, 587-593.

Șenol, G., Eriș, F.N., 2000. Haemophilus influenzae, Moraxella catarrhalis and Streptoccus pneumoniae strains isolated from respiratory infections and their resistance to antibiotics. Toraks Dergisi. 1, 46-50.

Tristram, S., Jacobs, M.R., Appelbaum, P.C., 2007. Antimicrobial Resistance in Haemophilus influenzae. Clin. Microbiol. Rev. 20, $368-389$.

Uncu, H., Colakoğlu, S., Turunç, T., Demiroğlu, Y.Z., Arslan, H., 2007. In vitro resistance rates of Streptococcus pneumoniae and Haemophilus influenzae clinical isolates to the antibiotics used in therapy. Mikrobiyol. Bul. 41, 441-446. 